\title{
Third-order superintegrable systems separable in parabolic coordinates
}

I. Popper, 1, a) S. Post, ${ }^{2, b}$ ) and P. Winternitz ${ }^{3,}$ c)

1) Le Département de Mathématiques et de Statistique, Université de Montréal. CP6128, Succursale Centre-Ville, Montréal (QC) H3C 3J\%,

Canada

${ }^{2)}$ Centre de Recherches Mathématiques, Université de Montréal. CP6128, Succursale Centre-Ville, Montréal (QC) H3C 3J\%, Canada

3) Le Département de Mathématiques et de Statistique and Centre de Recherches Mathématiques, Université de Montréal. CP6128, Succursale Centre-Ville, Montréal (QC) H3C 3J\%, Canada

In this paper, we investigate superintegrable systems which separate in parabolic coordinates and admit a third-order integral of motion. We give the corresponding determining equations and show that all such systems are multi-separable and so admit two second-order integrals. The third-order integral is their Lie or Poisson commutator. We discuss how this situation is different from the Cartesian and polar cases where new potentials were discovered which are not multi-separable and which are expressed in terms of Painlevé transcendents or elliptic functions.

PACS numbers: $02.30 . \mathrm{Ik}$ 45.20.Jj

Keywords: Integrability, Superintegrability, Separation of Variables, Classical and Quantum Mechanics

\footnotetext{
a) Electronic mail: popperi@DMS.umontreal.ca

b) Electronic mail: post@CRM.umontreal.ca

c) Electronic mail: wintern@CRM.umontreal.ca
} 


\section{INTRODUCTION}

This article is part of a research program the aim of which is to identify all third-order superintegrable systems in two-dimensional Euclidean space. We recall that a superintegrable system is one that has more integrals of motion than degrees of freedom. We consider a classical or quantum Hamiltonian

$$
H=\frac{1}{2}\left(p_{1}^{2}+p_{2}^{2}\right)+V\left(x_{1}, x_{2}\right)
$$

with two integrals of motion

$$
X_{a}=\sum_{0 \leq j+k \leq n} f_{a, j k}\left(x_{1}, x_{2}\right) p_{1}^{j} p_{2}, \quad a=1,2,
$$

where $p_{1}, p_{2}$ are components of the momentum $\vec{p}$. In classical mechanics, the integrals $X_{1,2}$ Poisson commute with $H$, are well defined functions on phase space and the three functions $\left\{H, X_{1}, X_{2}\right\}$ are functionally independent. The functions $X_{1}$ and $X_{2}$ do not Poisson commute with each other; instead they generate a non-Abelian algebra, usually a polynomial one. In quantum mechanics, $H$ and $X_{a}$ are Hermitian operators in the enveloping algebra of the Heisenberg algebra (or some generalization of the enveloping algebra). The operators $X_{a}$ Lie commute with $H$, but not with each other. Instead of functional independence, we assume that $H, X_{1}$ and $X_{2}$ are algebraically independent. More specifically, we assume that no Jordan polynomial in the operators $H, X_{1}$ and $X_{2}$ vanishes. As indicated in (2), we assume that $X_{a}$ are polynomials in the momentum. The "order of superintegrability" is the highest order of these polynomials.

The best known superintegrable systems are the Kepler-Coulomb system $1, \frac{1}{7}$ with potential $V=\alpha / r$ and the harmonic oscillator $\underline{15}, 28$ with $V=\alpha r^{2}$. As a matter of fact, these are the only rotationally invariant superintegrable systems in $n$-dimensions $(n \geq 2)$ (in agreement with Bertrand's theorem $-2 \cdot 9$ ). Both of these systems are quadratically superintegrable in that the Laplace-Runge-Lenz vector for $V=\alpha / r$ and the Fradkin (or quadropole) tensor for $V=\alpha r^{2}$ are both second-order in the momenta.

Until recently, most studies of superintegrability concentrated on the second-order case $e^{6}, \frac{824}{23}, 38$. At least in two-dimensional spaces, second-order superintegrable systems are well understood. All such systems in Euclidean spaces, spaces of constant curvature, and spaces of non-constant curvature with at least two second-order Killing tensors (Darboux spaces) have been classified $\underline{16}-\underline{18}, 22$. 
Recently, infinite families of superintegrable systems with integrals of motion of arbitrary order have been discovered and investigated $, 19-21,23,26,29,30,32,34,35$.

A systematic search for third-order superintegrable systems was started in 2002, both in classical and quantum mechanics ${ }^{11}$. However, the first (to our knowledge), article on thirdorder integrals of motion was written considerably earlier by Drach $\underline{5}$. He considered the case of one third-order integral of motion (in addition to the Hamiltonian) in flat two-dimensional complex space in classical mechanics. He found 10 different complex potentials which allow a third-order integral. Later it was shown that 7 of them are actually quadratically superintegrable and the third-order integral is reducible, i.e. is the Poisson commutator of two second-order integrals 31,37 .

The articles in Ref.'s 10, 11 and 36 were devoted to superintegrable systems with one third-order and one first or second-order integral. A first-order integral in $E_{2}(\mathbb{R})$ exists only if the potential is translationally or rotationally invariant, i.e. $V=V(x)$ or $V=V(r)$. In the classical case, all such potentials are second-order superintegrable and hence known. In quantum mechanics, one class of new superintegrable potentials is obtained $\underline{10}$ and is expressed in terms of elliptical functions, e.g.

$$
V=\hbar^{2} \omega^{2} s n^{2}(\omega x, k)
$$

where $\omega$ and $k$ are constants and $\operatorname{sn}(\omega x, k)$ is a Jacobian elliptic function ${ }^{3}$. The existence of a second-order integral implies that $V\left(x_{1}, x_{2}\right)$ allows separation of variables in Cartesian, polar, parabolic or elliptic coordinates. Cartesian and polar coordinates were considered earlier $\underline{10}, 36$. The study provided a number of new superintegrable potentials in the classical and, much more interestingly, in the quantum case. Indeed, quantum integrable systems with higher-order integrals of motion can be quite different from classical ones; a fact first noticed by Hietarinta 2,13 . The case of quantum superintegrable systems is much richer than that of classical ones. Third-order superintegrability with separation of variables in the Schrödinger equation in Cartesian or polar coordinates lead to potentials expressed in terms of Painlevé transcendents $\left(P_{I}, P_{I I}\right.$ and $P_{I V}$ for Cartesian coordinates, $P_{V I}$ for polar ones). The potentials separable in Cartesian coordinates have been intensively studied in both classical and quantum mechanics ${ }^{25}-27$.

The purpose of this article is to find all superintegrable systems that allow (at least) one third-order integral and a second-order integral that leads to separation in parabolic 
coordinates.

\section{THE DETERMINING EQUATIONS}

Let us now assume that the Hamiltonian allows separation of variables in parabolic coordinates

$$
x_{1}=\frac{1}{2}\left(\xi^{2}-\eta^{2}\right), \quad x_{2}=\xi \eta
$$

The quantum mechanical Hamiltonian has the form

$$
\begin{array}{r}
H=-\frac{\hbar^{2}}{\xi^{2}+\eta^{2}}\left(\frac{\partial^{2}}{\partial \xi^{2}}+\frac{\partial^{2}}{\partial \eta^{2}}\right)+V(\xi, \eta), \\
V(\xi, \eta)=\frac{W_{1}(\xi)+W_{2}(\eta)}{\xi^{2}+\eta^{2}}
\end{array}
$$

and there exists a second-order integral of the form

$$
Y=L_{3} p_{2}+p_{2} L_{3}+\frac{1}{\xi^{2}+\eta^{2}}\left(\xi^{2} W_{2}(\eta)-\eta^{2} W_{1}(\xi)\right)
$$

with

$$
\begin{array}{r}
p_{1}=-i \hbar \frac{\partial}{\partial x_{1}}=\frac{-i \hbar}{\xi^{2}+\eta^{2}}\left(\xi \frac{\partial}{\partial \xi}-\eta \frac{\partial}{\partial \eta}\right) \\
p_{2}=-i \hbar \frac{\partial}{\partial x_{2}}=\frac{-i \hbar}{\xi^{2}+\eta^{2}}\left(\eta \frac{\partial}{\partial \xi}+\xi \frac{\partial}{\partial \eta}\right) \\
L_{3}=-i \hbar\left(x_{2} p_{1}-x_{1} p_{2}\right)=\frac{i \hbar}{2}\left(\xi \frac{\partial}{\partial \eta}-\eta \frac{\partial}{\partial \xi}\right) .
\end{array}
$$

A third-order integral will then have the form

$$
X=\sum_{j+k+\ell=3} A_{j k \ell}\left\{L_{3}^{j}, p_{1}^{k} p_{2}^{\ell}\right\}+\left\{g_{1}\left(x_{1}, x_{2}\right), p_{1}\right\}+\left\{g_{2}\left(x_{1}, x_{2}\right), p_{2}\right\}
$$

The brackets $\{$,$\} denote anti-commutators, A_{j k \ell}$ are real constants and the functions $V$, $g_{1} g_{2}$ obey the four partial differential equations presented in Ref. 11 (in Cartesian coordinates). Here we need the equations in parabolic coordinates. To rewrite them in parabolic coordinates, it is convenient to replace the unknown functions $g_{1}\left(x_{1}, x_{2}\right)$ and $g_{2}\left(x_{1}, x_{2}\right)$ by $G_{1}(\xi, \eta), G_{2}(\xi, \eta)$, putting

$$
g_{1}\left(x_{1}, x_{2}\right)=\frac{\xi G_{1}(\xi, \eta)-\eta G_{2}(\xi, \eta)}{\xi^{2}+\eta^{2}}, \quad g_{2}\left(x_{1}, x_{2}\right)=\frac{\eta G_{1}(\xi, \eta)+\xi G_{2}(\xi, \eta)}{\xi^{2}+\eta^{2}}
$$


The four determining equations for the integral (7), i.e. the commutativity condition $[H, X]=0$, can be written as

$$
\begin{gathered}
G_{1, \xi}-\frac{\xi G_{1}-\eta G_{2}}{\xi^{2}+\eta^{2}}=h_{1}(\xi, \eta), \\
G_{1, \eta}+G_{2, \xi}-2 \frac{\eta G_{1}+\xi G_{2}}{\xi^{2}+\eta^{2}}=h_{2}(\xi, \eta), \\
G_{2, \eta}+\frac{\xi G_{1}-\eta G_{2}}{\xi^{2}+\eta^{2}}=h_{3}(\xi, \eta), \\
G_{1} V_{\xi}+G_{2} V_{\eta}=\frac{\hbar^{2}}{4} \phi,
\end{gathered}
$$

with

$$
\begin{aligned}
& h_{1}(\xi, \eta)=\frac{3 F_{1} V_{\xi}+F_{2} V_{\eta}}{\xi^{2}+\eta^{2}} \\
& h_{2}(\xi, \eta)=\frac{2 F_{2} V_{\xi}+2 F_{3} V_{\eta}}{\xi^{2}+\eta^{2}} \\
& h_{3}(\xi, \eta)=\frac{F_{3} V_{\xi}+3 F_{4} V_{\eta}}{\xi^{2}+\eta^{2}}
\end{aligned}
$$

and

$$
\begin{aligned}
\phi= & \frac{F_{1} V_{\xi \xi \xi}+F_{2} V_{\xi \xi \eta}+F_{3} V_{\xi \eta \eta}+F_{4} V_{\eta \eta \eta}}{\left(\xi^{2}+\eta^{2}\right)^{2}} \\
& +\frac{\left(3 \xi F_{1}+2 \eta F_{2}-\xi F_{3}\right) V_{\xi \xi}+\left(\eta F_{2}-\xi F_{2}-\eta F_{3}+\xi F_{4}\right) V_{\xi, \eta}+\left(\eta F_{2}-2 \xi F_{3}-3 \eta F_{4}\right) V_{\eta \eta}}{\left(\xi^{2}+\eta^{2}\right)^{3}} \\
& -\left(\frac{3\left(\xi^{2}-\eta^{2}\right)\left(F_{1}-F_{3}\right)+6 \xi \eta\left(F_{2}-F_{4}\right)}{\left(\xi^{2}+\eta^{2}\right)^{4}}-4 A_{300} \eta\left(\xi^{2}+\eta^{2}\right)+2 A_{201}(\xi+\eta)\right) V_{\xi} \\
& -\left(\frac{6 \xi \eta\left(F_{1}-F_{3}\right)-3\left(\xi^{2}-\eta^{2}\right)\left(F_{2}-F_{4}\right)}{\left(\xi^{2}+\eta^{2}\right)^{4}}-4 A_{300} \xi\left(\xi^{2}+\eta^{2}\right)-2 A_{201}(\xi+\eta)\right) V_{\eta} .
\end{aligned}
$$

The subscripts denote partial derivatives and the expressions $F_{1}, F_{2}, F_{3}, F_{4}$ are polynomials in $\xi$ and $\eta$ :

$$
\begin{aligned}
F_{1}= & \eta^{3} A_{003}+\xi \eta^{2} A_{012}+\xi^{3} A_{030}-\frac{1}{2} \eta^{3}\left(\xi^{2}+\eta^{2}\right) A_{102}-\frac{1}{2} \xi \eta^{2}\left(\xi^{2}+\eta^{2}\right) A_{111}-\frac{1}{2} \eta \xi^{2}\left(\xi^{2}+\eta^{2}\right) A_{120} \\
+ & \frac{1}{4} \eta^{3}\left(\xi^{2}+\eta^{2}\right)^{2} A_{201}+\frac{1}{4} \xi \eta^{2}\left(\xi^{2}+\eta^{2}\right)^{2} A_{210}-\frac{1}{8} \eta^{3}\left(\xi^{2}+\eta^{2}\right)^{3} A_{300}+\xi^{2} \eta A_{021} \\
F_{2}= & 3 \xi \eta^{2} A_{003}-\eta\left(\eta^{2}-2 \xi^{2}\right) A_{012}-3 \xi^{2} \eta A_{030}-\frac{1}{2} \xi \eta^{2}\left(\xi^{2}+\eta^{2}\right) A_{102}+\frac{1}{2} \eta^{3}\left(\xi^{2}+\eta^{2}\right) A_{111} \\
& +\frac{1}{2} \xi\left(\xi^{2}+2 \eta^{2}\right)\left(\xi^{2}+\eta^{2}\right) A_{120}-\frac{1}{4} \xi \eta^{2}\left(\xi^{2}+\eta^{2}\right)^{2} A_{201}-\frac{1}{4} \eta\left(\eta^{2}+2 \xi^{2}\right)\left(\xi^{2}+\eta^{2}\right)^{2} A_{210} \\
& +\frac{3}{8} \xi \eta^{2}\left(\xi^{2}+\eta^{2}\right)^{3} A_{300}-A_{021} \xi\left(2 \eta^{2}-\xi^{2}\right)
\end{aligned}
$$




$$
\begin{aligned}
F_{3}= & 3 \xi^{2} \eta A_{003}-\xi\left(2 \eta^{2}-\xi^{2}\right) A_{012}+3 \xi \eta^{2} A_{030}+\frac{1}{2} \eta \xi^{2}\left(\xi^{2}+\eta^{2}\right) A_{102}+\frac{1}{2} \xi^{3}\left(\xi^{2}+\eta^{2}\right) A_{111} \\
& -\frac{1}{2} \eta\left(\eta^{2}+2 \xi^{2}\right)\left(\xi^{2}+\eta^{2}\right) A_{120}-\frac{1}{4} \eta \xi^{2}\left(\xi^{2}+\eta^{2}\right)^{2} A_{201}+\frac{1}{4} \xi\left(\xi^{2}+2 \eta^{2}\right)\left(\xi^{2}+\eta^{2}\right)^{2} A_{210} \\
& -\frac{3}{8} \eta \xi^{2}\left(\xi^{2}+\eta^{2}\right)^{3} A_{300}+\eta A_{021}\left(\eta^{2}-2 \xi^{2}\right) \\
F_{4}= & \xi^{3} A_{003}-\xi^{2} \eta A_{012}-\eta^{3} A_{030}+\frac{1}{2} \xi^{3}\left(\xi^{2}+\eta^{2}\right) A_{102}-\frac{1}{2} \eta \xi^{2}\left(\xi^{2}+\eta^{2}\right) A_{111}+\frac{1}{2} \xi \eta^{2}\left(\xi^{2}+\eta^{2}\right) A_{120} \\
+ & \frac{1}{4} \xi^{3}\left(\xi^{2}+\eta^{2}\right)^{2} A_{201}-\frac{1}{4} \eta \xi^{2}\left(\xi^{2}+\eta^{2}\right)^{2} A_{210}+\frac{1}{8} \xi^{3}\left(\xi^{2}+\eta^{2}\right)^{3} A_{300}+\xi \eta^{2} A_{021} .
\end{aligned}
$$

The determining equations (8, 10) are the same in classical and quantum mechanics but (11) contains the Planck constant on the right hand side. The corresponding equation in classical mechanics is obtained by taking the limit $\hbar \rightarrow 0$ so (11) is greatly simplified. Hence the difference between classical and quantum integrability (and superintegrability) for third-order integrals of motion.

The system (8, 11) is overdetermined. The first three equations imply a linear compatibility condition for the potential

$$
\begin{aligned}
0= & F_{3} V_{\xi \xi \xi}+\left(3 F_{4}-2 F_{2}\right) V_{\xi \xi \eta}+\left(3 F_{1}-2 F_{3}\right) V_{\xi \eta \eta}+F_{2} V_{\eta \eta \eta} \\
& +\left(2\left(F_{3 \xi}-F_{2 \eta}\right)-\frac{3 \xi F_{1}-6 \eta F_{2}+7 \xi F_{3}}{\xi^{2}+\eta^{2}}\right) V_{\xi \xi}+\left(2\left(F_{2 \eta}-F_{3 \xi}\right)-\frac{3 \eta F_{4}-6 \xi F_{3}+7 \eta F_{2}}{\xi^{2}+\eta^{2}}\right) V_{\eta \eta} \\
& +\left(2\left(3 F_{1 \eta}-F_{2 \xi}-F_{3 \eta}+3 F_{4 \xi}\right)-\frac{21 \eta F_{1}-5 \eta F_{3}-5 \xi F_{2}+21 \xi F_{4}}{\xi^{2}+\eta^{2}}\right) V_{\xi \eta} \\
& +A V_{\eta}+B V_{\xi}
\end{aligned}
$$

where

$$
\begin{aligned}
A= & F_{2 \eta \eta}-2 F_{3 \eta \xi}+3 F_{4 \xi \xi}+\frac{-7 \eta F_{2 \eta}-\xi F_{2 \xi}+6 \xi F_{3 \eta}+6 \eta F_{3 \xi}-3 \eta F_{4 \eta}-21 \xi F_{4 \xi}}{\xi^{2}+\eta^{2}} \\
& +2 \frac{21 \xi^{2} F_{4}+F_{2} \xi^{2}+7 \eta^{2} F_{2}-12 \xi \eta F_{3}+3 F_{4} \eta^{2}}{\left(\xi^{2}+\eta^{2}\right)^{2}} \\
B= & 3 F_{1 \eta \eta}-2 F_{2 \eta \xi}+F_{3 \xi, \xi}-\frac{21 \eta F_{1 \eta}+3 \xi F_{1 \xi}-6 \xi F_{2 \eta}-6 \eta F_{2 \xi}+\eta F_{3 \eta}+7 \xi F_{3 \xi}}{\xi^{2}+\eta^{2}} \\
& +2 \frac{F_{3} \eta^{2}+3 F_{1} \xi^{2}+21 \eta^{2} F_{1}-12 \xi \eta F_{2}+7 \xi^{2} F_{3}}{\left(\xi^{2}+\eta^{2}\right)^{2}} .
\end{aligned}
$$

Compatibility between the first three determining equations (8, 10] and the fourth one (11) requires three more conditions, this time nonlinear ones. Indeed, solving (11) for $G_{2}$, we have

$$
G_{2}=\frac{1}{V_{\eta}}\left(\frac{\hbar^{2}}{4} \Phi-V_{\xi} G_{1}\right), \quad V_{\eta} \neq 0
$$


Replacing $G_{2}$ from (14) into (8, [10), the system can then be solved for $G_{1}$,

$$
G_{1}=\frac{h_{4}}{h_{5}}, \quad G_{2}=\frac{h_{5} \hbar^{2} \Phi-4 V_{\xi} h_{4}}{4 h_{5} V_{\eta}}, \quad h_{5} \neq 0,
$$

with

$$
\begin{aligned}
h_{4}= & \left(\xi^{2}+\eta^{2}\right)\left(4 h_{3} V_{\eta}^{3}+4 h_{2} V_{\xi} V_{\eta}^{2}+4 h_{1} V_{\xi}^{2} V_{\eta}-\hbar^{2} V_{\eta}^{2} \Phi_{\eta}-\hbar^{2} V_{\xi} V_{\eta} \Phi_{\xi}-\hbar^{2}\left(V_{\eta \eta} V_{\eta}-V_{\xi \eta} V_{\xi}\right) \Phi\right) \\
& +4 \eta V_{\xi}^{2}-4 \eta V_{\eta}^{2}-8 \xi V_{\eta} V_{\xi} \\
h_{5}= & 4\left(\xi^{2}+\eta^{2}\right)\left(V_{\xi} V_{\eta}\left(V_{\xi \xi}-V_{\eta \eta}+\left(V_{\eta}^{2}-V_{\xi}^{2}\right) V_{\xi \eta}\right)+4\left(\eta V_{\xi}-\xi V_{\eta}\right)\left(V_{\xi}^{2}+V_{\eta}^{2}\right) .\right.
\end{aligned}
$$

Replacing (15), into (8, 10), gives the three additional non-linear compatibility conditions on the potential, namely

$$
\begin{array}{r}
\left(\frac{h_{4}}{h_{5}}\right)_{\xi}+\frac{\eta \hbar^{2} h_{5} \Phi+4 h_{4}\left(\xi V_{\eta}+\eta V_{\xi}\right)}{4 h_{5} V_{\eta}\left(\xi^{2}+\eta^{2}\right)}=h_{1} \\
\left(\frac{h_{4}}{h_{5}}\right)_{\eta}+\left(\frac{\hbar^{2} h_{5} \Phi-4 V_{\xi} h_{4}}{4 h_{5} V_{\eta}}\right)_{\xi}+\frac{\xi \hbar^{2} h_{5} \Phi+4 h_{4}\left(\eta V_{\eta}-\xi V_{\xi}\right)}{2 h_{5} V_{\eta}\left(\xi^{2}+\eta^{2}\right)}=h_{2} \\
\left(\frac{\hbar^{2} h_{5} \Phi-4 V_{\xi} h_{4}}{4 h_{5} V_{\eta}}\right)_{\eta}-\frac{\eta \hbar^{2} h_{5} \Phi+4 h_{4}\left(\xi V_{\eta}+\eta V_{\xi}\right)}{4 h_{5} V_{\eta}\left(\xi^{2}+\eta^{2}\right)}=h_{3} .
\end{array}
$$

\section{GENERAL FORMS FOR $W_{1}$ AND $W_{2}$}

In order to determine all possible potentials which separate in parabolic coordinates and admit a third-order integral of motion, we begin with the linear compatibility condition (13). Replacing $V$ with the form as in (5), the compatibility condition can be differentiated to obtain a system of linear ordinary differential equations (ODEs) for $W_{1}$ and $W_{2}$. These equations are given in Appendix $\mathrm{A}$ for $W_{1}$. Notice that interchanging $\xi$ and $\eta$ has the effect of changing the sign of the coefficients to $(-1)^{j+k} A_{j k \ell}$. Thus, equations for $W_{2}$ are of the same form as (A1,A14), up to a change in sign in these constants. We begin our search for the admissible potentials by solving equations (A1,A14).

Theorem 1 Given a Hamiltonian which admits a third-order integral of motion with a potential which separates in parabolic coordinates as in (5). Then any admissible terms in the potential are included in

$$
W_{1}(\xi)=\sum_{j=0}^{16} c_{j} \xi^{j}+c_{-2} \xi^{-2}+c_{-4} \xi^{-4}+c_{-6} \xi^{-6}+\frac{\alpha_{1} \xi \ln \left(\xi+\sqrt{\xi^{2}+B_{1}}\right)+\alpha_{2} \xi}{\sqrt{\xi^{2}+B_{1}}}
$$




$$
W_{2}(\eta)=\sum_{j=1}^{16} d_{j} \eta^{j}+d_{-2} \eta^{-2}+d_{-4} \eta^{-4}+d_{-6} \eta^{-6}+\frac{\beta_{1} \ln \left(\eta+\sqrt{\eta^{2}+B_{2}}\right)+\beta_{2} \eta}{\sqrt{\eta^{2}+B_{2}}} .
$$

In particular, any solution of the system (13) has the form (5) with $W_{1}$ and $W_{2}$ as in (19) and (20) with appropriately chosen constants.

Proof: Beginning with (A1), in the case that $A_{300} \neq 0$, the solutions are given by

$$
W_{1}=\sum_{i=0}^{14} c_{i} \xi^{i}+c_{-2} \xi^{-2}+c_{-4} \xi^{-4} .
$$

Similarly, for (즈), in the case that $A_{210} \neq 0$, the solutions are

$$
W_{1}=\sum_{i=0}^{15} c_{i} \xi^{i}+c_{-2} \xi^{-2}
$$

Equation (A3) in addition requires that $c_{14}=c_{15}=0$.

Now assume $A_{300}=A_{210}=0$. This sets (A1,A3) to be satisfied identically and (A4) becomes

$$
-\left(\left(\xi^{2} A_{201}+2 A_{120}\right) \frac{d^{17}}{d \xi^{17}}+33 \xi A_{201} \frac{d^{16}}{d \xi^{16}}+255 A_{201} \frac{d^{15}}{d \xi^{15}}\right) W_{1}=0 .
$$

The solutions of (23), assuming $A_{201} \neq 0$, are

$$
W_{1}=\sum_{i=0}^{14} c_{i} \xi^{i}+\frac{\alpha_{1} \xi \ln \left(\xi+\sqrt{\xi^{2}+B_{1}}\right)+\alpha_{2} \xi}{\sqrt{\xi^{2}+B_{1}}} .
$$

with $A_{120}=B_{1} A_{201} / 2$. If $A_{201}=0$, the solutions are

$$
W_{1}=\sum_{i=0}^{16} c_{i} \xi^{i}
$$

Now assume $A_{300}=A_{210}=A_{201}=A_{120}=0$, (A1 A4) are then identically satisfied and (A5) becomes

$$
\frac{1}{2}\left(\left(A_{102} \xi^{2}+2 A_{021}\right) \frac{d^{17}}{d \xi^{17}}+255 A_{102} \frac{d^{15}}{d \xi^{15}}+33 \xi A_{102} \frac{d^{16}}{d \xi^{16}}\right) W_{1}=0 .
$$

The solutions of (26) for $A_{102} \neq 0$ are as (24) with $A_{021}=2 A_{102} B_{1}$ and for $A_{102}=0$ are as (25).

If we now assume $A_{300}=A_{210}=A_{201}=A_{120}=A_{102}=A_{021}=0$, (A6) becomes

$$
A_{003}\left[3 \xi^{2} \frac{d^{17}}{d \xi^{17}}+111 \xi \frac{d^{16}}{d \xi^{16}}+969 \frac{d^{15}}{d \xi^{15}}\right] W_{1}=0,
$$


the solutions of which are given by (21).

Now, assume $A_{300}=A_{210}=A_{201}=A_{120}=A_{102}=A_{021}=A_{003}=0$, this implies (A1. A6) as well as (A10 A12) are identically satisfied. Equation (A7) becomes

$$
\begin{aligned}
\frac{\xi\left(A_{111} \xi^{2}+6 A_{030}-4 A_{012}\right)}{2} \frac{d^{17}}{d \xi^{17}} W_{1} & +\left(\frac{51}{2} A_{111} \xi^{2}-36 A_{12}+54 A_{030}\right) \frac{d^{16}}{d \xi^{16}} W_{1} \\
+ & \frac{813 \xi A_{111}}{2} \frac{d^{15}}{d \xi^{15}} W_{1}+2016 A_{111} \frac{d^{14}}{d \xi^{14}} W_{1}=0 .
\end{aligned}
$$

The general solutions of (28) are of the form

$$
W_{1}=\sum_{i=0}^{13} c_{i} \xi^{i}+c_{-2} \xi^{-2}+\frac{\alpha_{1} \xi \ln \left(\xi+\sqrt{\xi^{2}+B_{1}}\right)+\alpha_{2} \xi}{\sqrt{\xi^{2}+B_{1}}}
$$

with $A_{030}=\frac{1}{6} B_{1} A_{111}+\frac{2}{3} A_{012}$ for $A_{111} \neq 0$ and of the form (22) for $A_{111}=0$.

Now assume that $A_{300}=A_{210}=A_{201}=A_{120}=A_{102}=A_{021}=A_{003}=A_{111}=3 A_{030}-$ $2 A_{012}=0$, this implies that (A14) has the form

$$
A_{012}\left(\xi^{3} \frac{d^{17}}{d \xi^{17}}+57 \xi^{2} \frac{d^{16}}{d \xi^{16}}+1023 \xi \frac{d^{15}}{d \xi^{15}}+5760 \frac{d^{14}}{d \xi^{14}}\right) W_{1}=0 .
$$

The solutions of (30) for $A_{012} \neq 0$ are given by

$$
W_{1}=\sum_{i=0}^{14} c_{i} \xi^{i}+c_{-2} \xi^{-2}+c_{-4} \xi^{-4}+c_{-6} \xi^{-6}
$$

This is the final case, since assuming $A_{012}=0$ in addition to $A_{300}=A_{210}=A_{201}=A_{120}=$ $A_{102}=A_{021}=A_{003}=A_{111}=3 A_{030}-2 A_{012}=0$ has all the A's equal 0 and so there is no longer a third order term in our constant of motion.

Thus, the most general form of the function $W_{1}$ is given by (19). By direct analogy, the most general form of the function $W_{2}$ is given by (20) .

Corollary 1 The potential for any 3rd-order superintegrable system which separates in parabolic coordinates satisfies a non-trivial system of linear ODEs for both $W_{1}$ and $W_{2}$.

Proof As shown above, the compatibility conditions (13) are satisfied identically if and only if all the $A_{i j k}$ are 0 . On the other hand, if there is a non-zero $A_{i j k}$ then the functions $W_{1}$ and $W_{2}$ in the potential will satisfy some linear ODEs. 


\section{THE ABSENCE OF IRRATIONAL TERMS}

In this section, we show that the only possible form of the potential is as a rational function of $\xi$ and $\eta$. We shall show this by contradiction. Namely, we consider the case that either $\alpha_{1} \neq 0$ or both $\alpha_{2} \neq 0$ and $B_{1} \neq 0$ in (19).

If we substitute the general form (19) into equations (A1,A14), it is immediate from the previous section that $A_{300}=A_{210}=0$. We also obtain the following possible restrictions on the constants, as suggested in the previous section:

$$
\left\{\begin{array}{c}
A_{003}=A_{021}=\frac{B_{1}\left(A_{102}-A_{120}\right)}{2}, \\
A_{012}=A_{030}=\frac{A_{111} B_{1}}{2}, \\
A_{201}=\frac{2 A_{120}}{B_{1}} .
\end{array}\right\}
$$

In the case that $B_{1} \neq 0, W_{1}$ becomes

$$
W_{1}=c_{0}+c_{1} \xi+c_{2} \xi^{2}+c_{3} \xi^{3}+c_{4} \xi^{4}+c_{5} \xi^{5}+c_{6} \xi^{6}+\frac{c_{-2}}{\xi^{2}}+\frac{\alpha_{1} \xi \ln \left(\xi+\sqrt{\xi^{2}+B_{1}}\right)+\alpha_{2} \xi}{\sqrt{\xi^{2}+B_{1}}}
$$

with the following cases

$$
\begin{aligned}
& \left\{B_{1} \neq 0, c_{-2}=c_{5}=c_{6}=0\right\} \\
& \left\{B_{1} \neq 0, A_{201}=0, c_{-2}=c_{6}=0\right\} \\
& \left\{B_{1} \neq 0, A_{111}=A_{201}=0, c_{-2}=0\right\} \\
& \left\{B_{1} \neq 0, A_{102}=A_{201}=0 .\right\}
\end{aligned}
$$

In the case that $\alpha_{1} \neq 0$ and $B_{1}=0, W_{1}$ becomes

$$
W_{1}=c_{0}+c_{1} \xi+c_{2} \xi^{2}+c_{3} \xi^{3}+c_{4} \xi^{4}+c_{5} \xi^{5}+c_{6} \xi^{6}+\frac{c_{-2}}{\xi^{2}}+\frac{c_{-4}}{\xi^{2}}+\alpha_{1} \ln (\xi),
$$

with the following cases

$$
\begin{aligned}
& \left\{\alpha_{1} \neq 0, B_{1}=0, c_{-4}=c_{5}=c_{6}=0\right\} \\
& \left\{\alpha_{1} \neq 0, B_{1}=0, A_{201}=0, c_{-4}=c_{6}=0\right\} \\
& \left\{\alpha_{1} \neq 0, B_{1}=0, A_{111}=A_{201}=0, c_{-4}=0\right\} \\
& \left\{\alpha_{1} \neq 0, B_{1}=0, A_{102}=A_{201}=0\right\} .
\end{aligned}
$$

Note that when $B_{1}=0, \alpha_{2}$ becomes an additive constant which is absorbed into $c_{0}$.

Next, we substitute these cases (34) or (36), along with the forms of $W_{1}$ (33) or (35) and $W_{2}$ (20), into the compatibility condition (13) to obtain

$$
0=M_{2} \ln \left(\eta^{2}+\sqrt{\eta^{2}+B_{2}}\right)+M_{1} \sqrt{\eta^{2}+B_{2}}+M_{0}
$$


where the $M_{i}$ are rational functions which are too long to be presented here but are available from the authors upon request. Solving $M_{2}=0$ gives 3 possibilities: either $\beta_{1}=0, B_{2}=-B_{1}$ or, in the case that $B_{1}$ is not zero, $B_{2}$ may vanish if additionally $A_{111}=0$ and $A_{102}=$ $B_{1} A_{201} / 2$.

Solving $M_{1}=0$ and $M_{0}=0$ gives similar forms for $W_{2}$ : If $B_{1} \neq 0$, then $W_{2}$ has the form

$$
W_{2}=d_{1} \eta+c_{2} \eta^{2}-c_{4} \eta^{4}+c_{6} \eta^{6}+\frac{c_{-2}}{\eta^{2}}+\frac{-\alpha_{1} \eta \ln \left(\eta+\sqrt{\eta^{2}+B_{2}}\right)+\beta_{2} \eta}{\sqrt{\eta^{2}+B_{2}}}
$$

and if $B_{1}=0$, then it has the form

$$
W_{2}=c_{2} \eta^{2}-c_{4} \eta^{4}+c_{6} \eta^{6}+\frac{c_{-2}}{\eta^{2}}-\frac{c_{-4}}{\eta^{4}}-\alpha_{1} \ln (\eta)
$$

In both cases, $c_{3}$ and $c_{5}$ are also required to be 0 as well and as several additional sets of constraints which are required to completely solve (13). We shall return to some of these cases later.

To obtain a contradiction for the potentials admitting logarithmic singularities, we now turn our attention to the non-linear compatibility conditions. Beginning with Eq. (16), we clear the denominator and consider the equation

$$
0=h_{5}^{2} V_{\eta}\left(\xi^{2}+\eta^{2}\right)\left[\left(\frac{h_{4}}{h_{5}}\right)_{\xi}+\frac{\eta \hbar^{2} h_{5} \Phi+4 h_{4}\left(\xi V_{\eta}+\eta V_{\xi}\right)}{4 h_{5} V_{\eta}\left(\xi^{2}+\eta^{2}\right)}-h_{1}\right] .
$$

In the case that both $\alpha_{1}$ and $B_{1}$ are assumed non-zero, equation (40) will have polynomial dependence on the quantity $\ln \left(\xi^{2}+\sqrt{\xi^{2}+B_{1}}\right)$. Substituting the forms of the potential obtained above (33) and (38) into this quantity,(40), gives

$$
\begin{aligned}
0= & 32 \alpha^{6} B_{1}^{3} K \ln ^{6}\left(\xi^{2}+\sqrt{\xi^{2}+B_{1}}\right)+\mathcal{O}\left(\ln ^{5}\left(\xi^{2}+\sqrt{\xi^{2}+B_{1}}\right)\right) \\
K= & 9 A_{111} \eta \xi^{14}+\frac{9 A_{111}}{2}\left(16 \eta^{2}+5 B_{1}\right) \xi^{12} \\
& +\left(2\left(48 A_{102}-23 A_{201}\right) \eta^{2}-B_{1}\left(16 A_{102}-11 A_{201}\right)\right) \eta^{2} \xi^{11}+\mathcal{O}\left(\xi^{10}\right) .
\end{aligned}
$$

Therefore, since it was assumed that $\alpha_{1} \neq 0$ and $B_{1} \neq 0$, the condition (40) requires that $A_{111}=A_{102}=A_{201}=0$ which is a contradiction because in this case all of the $A_{j k \ell}$ 's would be identically zero. In the case that $B_{1}=0$, (40) is a polynomial in $\ln \xi$ with leading order term

$$
0=\frac{K}{\left(\xi^{2}+\eta^{2}\right)^{10} \xi^{4} \eta^{3}} \ln ^{4} \xi+\mathcal{O}\left(\ln ^{3} \xi\right)
$$




$$
K=192 \alpha_{1}^{6} A_{111} \xi^{12} \eta^{2}-640 \alpha_{1}^{6} A_{102} \xi^{11} \eta^{3}-32\left(864 d_{-2} c_{4} A_{102}+\alpha^{2} A_{201}\right) \alpha^{4} \xi^{13} \eta^{3}+\ldots
$$

For simplicity, we give only the most relevant terms instead of the highest order ones in $K$. From these terms, it can be seen that the condition (40) leads to a contradiction since they would imply $A_{201}, A_{102}$ and $A_{111}$ are all 0 and so every $A_{j k \ell}$ would vanish. Thus, there are no logarithmic singularities in the potential.

Next, we turn in particular to the case that $\alpha_{1}=\beta_{1}=0$. In this case, we proceed slightly differently because computationally, it is more difficult for MAPLE to compute coefficients of the expression (40) with respect to $\left(\xi^{2}+\eta^{2}\right)^{k / 2}$ without first simplifying the entire expression. On the other hand, unlike in the previous section, we can solve (8, 10) without much difficultly and replace the integrated forms of $G_{1}$ and $G_{2}$ into (11) to obtain the needed contradictions.

In this case, we require the complete solutions for (13). Namely, $W_{1}$ must satisfy (33) with $c_{3}=c_{5}=0$ and $W_{2}$ must satisfy (38), both with $\alpha_{1}=\beta_{1}=0$. Additionally, the constants satisfy one of the following cases:

$$
\begin{array}{r}
\left\{B_{2}=-B_{1}, A_{201}=A_{102}=0, c_{0}=c_{1}=d_{1}=c_{6}=0\right\} \\
\left\{B_{2}=-B_{1}, A_{201}=A_{111}=0, c_{0}=c_{1}=d_{1}=c_{-2}=0\right\} \\
\left\{B_{2}=-B_{1}, A_{111}=0, A_{102}=A_{120}, c_{1}=d_{1}=c_{6}=c_{-2}=0\right\} \\
\left\{B_{2}=0, A_{111}=0, A_{102}=A_{120}, c_{0}=c_{1}=d_{1}=c_{6}=c_{-2}=0\right\} \\
\left\{B_{2}=-B_{1}, c_{0}=c_{1}=d_{1}=c_{6}=c_{-2}=0\right\} .
\end{array}
$$

There is also a complex solution for the constants

$$
\left\{B_{2}=-B_{1}, A_{201}=0, A_{111}=i A_{102}, d_{1}=i c_{1}, c_{0}=c_{6}=c_{-2}=0\right\}
$$

To obtain the needed contradictions, we use the obtained sets of solutions for (13) to solve (8, 10) for $G_{1}$ and $G_{2}$ and replace these solutions into (11). For example, in the case identified in (45) the relevant $G^{\prime} s$ are

$$
\begin{aligned}
G_{1}= & \left(\frac{-\left(-3 B_{1} \xi^{2}+4 \xi^{2} \eta^{2}+\eta^{2} B_{1}\right) \alpha_{2}}{2 \sqrt{\xi^{2}+B_{1}}\left(\xi^{2}+\eta^{2}\right)}+\frac{\xi \eta\left(-\eta^{2}+2 B_{1}+\xi^{2}\right) \beta_{2}}{\sqrt{\eta^{2}-B_{1}}\left(\xi^{2}+\eta^{2}\right)}\right. \\
& \left.-1 / 2\left(2 \eta^{4}+\eta^{2} B_{1}+4 \xi^{2} \eta^{2}-3 B_{1} \xi^{2}\right) \xi c_{4}-\frac{\left(\eta^{2}-\xi^{2}-B_{1}\right) c_{-2}}{\eta^{2} \xi}\right) A_{111} \\
& +\frac{\eta\left(\xi^{2}+\eta^{2}\right)}{6} k_{1}+\eta k_{2}+\xi k_{3}
\end{aligned}
$$




$$
\begin{aligned}
G_{2}= & \left(-\frac{\xi \eta\left(-\eta^{2}+2 B_{1}+\xi^{2}\right) \alpha_{2}}{\sqrt{\xi^{2}+B_{1}}\left(\xi^{2}+\eta^{2}\right)}-1 / 2 \frac{\left(-B_{1} \xi^{2}+4 \xi^{2} \eta^{2}+3 \eta^{2} B_{1}\right) \beta_{2}}{\sqrt{\eta^{2}-B_{1}}\left(\xi^{2}+\eta^{2}\right)}\right. \\
& \left.+1 / 2 \eta\left(2 \xi^{4}-B_{1} \xi^{2}+4 \xi^{2} \eta^{2}+3 \eta^{2} B_{1}\right) c_{4}+\frac{\left(\eta^{2}-\xi^{2}-B_{1}\right) c_{-2}}{\xi^{2} \eta}\right) A_{111} \\
& -\frac{\xi\left(\xi^{2}+\eta^{2}\right)}{6} k_{1}-\eta k_{3}+\xi k_{2}
\end{aligned}
$$

where $k_{1}, k_{2}$ and $k_{3}$ are constants of integration. These solutions for $G_{1}$ and $G_{2}$ when substituted into (11) give

$$
0=T_{1} \sqrt{\xi^{2}+B_{1}} \sqrt{\eta^{2}-B_{1}}+T_{2} \sqrt{\xi^{2}+B_{1}}+T_{3} \sqrt{\eta^{2}-B_{1}}+T_{4}
$$

Again, the $T_{i}$ 's are rational functions of $\xi$ and $\eta$, and can be obtained from the authors. Solving these systems, we obtain $\beta_{2}=0$ and $\alpha_{2}=0$ or $A_{111=0}$, which gives a contradiction, since in these cases either all of the $A_{j k \ell}$ are zero or the potential reduces to a rational function. By checking each case in this manner, we find that when $B_{1}$ is assumed to be non-zero the potentials reduce to rational functions.

Thus, we have shown by contradiction that the only possible potentials which satisfy both the linear (13) and nonlinear (16,-18) compatibility conditions are rational functions of $\xi$ and $\eta$.

\section{FINAL LIST OF SUPERINTEGRABLE POTENTIALS}

Since there are no longer any irrational terms in the potential, it is a straightforward computation to find the admissible choices of constants which satisfy the linear compatibility condition (13), to use these choices to solve the linear partial differential equations (8-10) for $G_{1}$ and $G_{2}$ and to solve the resulting algebraic system determined by the coefficients of (11). In this section, we exhibit the possible potentials which remain, i.e. those potentials which separate in parabolic coordinates and admit a third order integral of motion. Remarkably, the only such potentials are second-order superintegrable. These are exactly the potentials which separate in parabolic coordinates as well as another orthogonal coordinate system in $E_{2}(\mathbb{R})$. We also obtain a potential in $E(1,1)$ which is presented in Appendix $\mathbb{B}$.

It is interesting to note that, in addition to the systems which admit a single thirdorder integral, this method allows us to obtain potentials in the quantum case which admit more than one third-order integral. Furthermore, these additional potentials give proof 
that the quantum correction to (11), namely $\Phi$, is not identically 0 for potentials which are superintegrable in both the classical and quantum cases. The quantity $\Phi$ only vanishes when, in addition, the appropriate choices of $A_{j k l}$ are assumed. For example, for potential $V_{1}$ below, the quantity $\Phi$ vanishes only when all of the $A_{j k l}$ 's are assumed 0 except for $A_{012}$.

\section{A. Potentials which admit a single third-order integral}

\section{A deformation of the anisotropic oscillator potential: $V_{1}$}

The following potential

$$
\begin{aligned}
V_{1} & =\left(\eta^{4}-\xi^{2} \eta^{2}+\xi^{4}\right) \alpha+\beta\left(\xi^{2}-\eta^{2}\right)+\frac{\gamma}{\xi^{2} \eta^{2}} \\
& =\alpha\left(4 x^{2}+y^{2}\right)+2 \beta x+\frac{\gamma}{y^{2}} \\
& =\alpha r^{2}\left(3 \cos ^{2} \theta+1\right)+2 \beta r \cos \theta+\frac{\gamma}{r^{2} \sin ^{2} \theta}
\end{aligned}
$$

admits a third-order integral with all all $A_{j k \ell}=0$ except $A_{021}$ and functions

$$
\begin{gathered}
G_{1}=2 \frac{A_{012}\left(-2 \alpha \xi^{2} \eta^{6}+\eta^{4} \alpha \xi^{4}+\eta^{4} \beta \xi^{2}+\gamma\right)}{\xi \eta^{2}} \\
G_{2}=-2 \frac{A_{012}\left(-2 \eta^{2} \alpha \xi^{6}+\eta^{4} \alpha \xi^{4}-\eta^{2} \beta \xi^{4}+\gamma\right)}{\eta \xi^{2}} .
\end{gathered}
$$

\section{A deformation of the Coulomb potential: $V_{2}$}

The following potential

$$
\begin{aligned}
V_{2} & =\frac{1}{\xi^{2}+\eta^{2}}\left(\frac{\alpha}{\xi^{2}}+\frac{\beta}{\eta^{2}}+\gamma\right) \\
& =\frac{1}{2 \sqrt{x^{2}+y^{2}}}\left(\frac{\alpha}{x+\sqrt{x^{2}+y^{2}}}+\frac{\beta}{x-\sqrt{x^{2}+y^{2}}}+\gamma\right) \\
& =\frac{\alpha}{2 r^{2}(\cos \theta+1)}-\frac{\beta}{2 r^{2}(\cos \theta-1)}+\frac{\gamma}{2 r}
\end{aligned}
$$

admits one third-order constant of motion with all $A_{j k \ell}=0$ except $A_{210}$ and functions

$$
\begin{gathered}
G_{1}=\frac{A_{210}\left(2 \eta^{4} \gamma \xi^{2}+2 \eta^{4} \alpha+4 \xi^{2} \beta \eta^{2}-\eta^{2} \xi^{2} h^{2}+2 \xi^{4} \beta\right)}{4 \xi \eta^{2}} \\
G_{2}=-\frac{A_{210}\left(2 \xi^{4} \beta+2 \xi^{4} \gamma \eta^{2}+4 \xi^{2} \alpha \eta^{2}-\eta^{2} \xi^{2} h^{2}+2 \eta^{4} \alpha\right)}{4 \eta \xi^{2}} .
\end{gathered}
$$




\section{A second deformation of the Coulomb potential: $V_{3}$}

The following potential

$$
\begin{aligned}
V_{3} & =\frac{\alpha \xi+\beta \eta+\gamma}{\xi^{2}+\eta^{2}} \\
& =\frac{1}{2 \sqrt{x^{2}+y^{2}}}\left(\alpha \sqrt{x+\sqrt{x^{2}+y^{2}}}+\beta \sqrt{\sqrt{x^{2}+y^{2}}-x}+\gamma\right) \\
& =\frac{1}{2 r}\left(\alpha \sqrt{2} \cos \frac{\theta}{2}+\beta \sqrt{2} \sin \frac{\theta}{2}+\gamma\right)
\end{aligned}
$$

admits one third-order integral associated with $A_{102}$ (all the remaining $A_{j k \ell}=0$ )

$$
\begin{gathered}
G_{1}=-\frac{1}{2} A_{102}\left(-\beta \xi^{2}+\beta \eta^{2}+2 \eta \alpha \xi+2 \eta \gamma\right) \\
G_{2}=\frac{1}{2} A_{102}\left(\alpha \xi^{2}-\alpha \eta^{2}+2 \xi \gamma+2 \eta \xi \beta\right) .
\end{gathered}
$$

\section{B. Potentials which admit more than one third-order integrals}

The following potentials are sub-cases of those from the previous section, in the quantum cases. They admit at least two third-order integrals.

\section{1. $V_{1}$ subcases}

The following potential

$$
\begin{aligned}
V_{1, a} & =\beta\left(\xi^{2}-\eta^{2}\right)+\frac{h^{2}}{\xi^{2} \eta^{2}} \\
& =2 \beta x+\frac{\hbar^{2}}{y^{2}} .
\end{aligned}
$$

admits three linearly independent third-order integrals associated with the constants $A_{012}, A_{003}$ and $A_{102}$ (the remaining $A_{j k \ell}$ 's are 0 ). The functions $G_{1}$ and $G_{2}$ are

$$
\begin{gathered}
G_{1}=-\frac{\left(3 h^{2} \eta^{2}-h^{2} \xi^{2}+2 \eta^{4} \xi^{4} \beta\right) A_{102}}{2 \eta \xi^{2}}+3 \frac{h^{2} A_{003}}{\eta \xi^{2}}+2 \frac{\left(\eta^{4} \xi^{2} \beta+h^{2}\right) A_{012}}{\xi \eta^{2}} \\
G_{2}=-\frac{\left(-3 h^{2} \xi^{2}+h^{2} \eta^{2}+2 \xi^{4} \eta^{4} \beta\right) A_{102}}{2 \xi \eta^{2}}+3 \frac{h^{2} A_{003}}{\xi \eta^{2}}+2 \frac{\left(\xi^{4} \eta^{2} \beta-h^{2}\right) A_{012}}{\eta \xi^{2}} .
\end{gathered}
$$




\section{2. $V_{2}$ subcases}

The following potential

$$
\begin{aligned}
V_{2, a} & =\frac{\gamma}{\xi^{2}+\eta^{2}}+\frac{h^{2}}{\xi^{2} \eta^{2}} \\
& =\frac{\gamma}{2 r}+\frac{\hbar^{2}}{r^{2} \sin ^{2} \theta} .
\end{aligned}
$$

admits two linearly independent third-order integrals associated with the constants $A_{300}$ and $A_{210}$ (the remaining $A_{j k \ell}$ 's are 0 ). The functions $G_{1}$ and $G_{2}$ are

$$
\begin{gathered}
G_{1}=-\frac{h^{2}\left(\xi^{2}+\eta^{2}\right)\left(3 \xi^{4}+2 \xi^{2} \eta^{2}+3 \eta^{4}\right) A_{300}}{8 \eta \xi^{2}}+\frac{\left(3 \eta^{2} \xi^{2} h^{2}+2 \eta^{4} \gamma, \xi^{2}+2 \eta^{4} h^{2}+2 \xi^{4} h^{2}\right) A_{210}}{4 \eta^{2} \xi} \\
G_{2}=\frac{h^{2}\left(\xi^{2}+\eta^{2}\right)\left(3 \xi^{4}+2 \xi^{2} \eta^{2}+3 \eta^{4}\right) A_{300}}{8 \xi \eta^{2}}-\frac{\left(3 \eta^{2} \xi^{2} h^{2}+2 \xi^{4} h^{2}+2 \eta^{2} \xi^{4} \gamma+2 \eta^{4} h^{2}\right) A_{210}}{4 \eta \xi^{2}} .
\end{gathered}
$$

Another subcase of the potential $V_{2}$ which admits three linearly independent third-order integrals is given by

$$
\begin{aligned}
V_{2, b} & =\frac{1}{\xi^{2}+\eta^{2}}\left(\gamma+\frac{h^{2}}{\xi^{2}}\right) \\
& =\frac{\gamma}{2 r}+\frac{\hbar^{2}}{2 r^{2}(1+\cos \theta)} .
\end{aligned}
$$

The integrals are associated with coefficients $A_{300}, A_{210}$ and $A_{201}$ with the remaining $A_{j k \ell}=0$ and functions

$$
\begin{aligned}
G_{1}= & \frac{h^{2} \eta\left(3 \eta^{2}+2 \xi^{2}\right)\left(\xi^{2}+\eta^{2}\right) A_{300}}{8 \xi^{2}}+\frac{\left(2 h^{2} \eta^{2}+2 \eta^{2} \xi^{2} \gamma-h^{2} \xi^{2}\right) A_{210}}{4 \xi} \\
& -\frac{A_{201} \eta\left(2 \xi^{4} \gamma-3 h^{2} \eta^{2}\right)}{4 \xi^{2}} \\
G_{2}= & \frac{h^{2}\left(3 \eta^{2}+2 \xi^{2}\right)\left(\xi^{2}+\eta^{2}\right) A_{300}}{8 \xi}-\frac{\eta\left(2 \xi^{4} \gamma+2 h^{2} \eta^{2}+3 h^{2} \xi^{2}\right) A_{210}}{4 \xi^{2}} \\
& +\frac{A_{201}\left(2 \xi^{4} \gamma-h^{2} \eta^{2}\right)}{4 \xi} .
\end{aligned}
$$

\section{Potentials admitting group symmetry}

The following potentials admit a Killing vector associated with group symmetry.

\section{Rotational symmetry}

The Coulomb potential

$$
V_{C}=\frac{\alpha}{\xi^{2}+\eta^{2}}=\frac{\alpha}{r}
$$


admits four linearly independent third-order integrals associated with constants $A_{300}, A_{210}, A_{201}$ and $A_{102}$ as well as a Killing vector associated with $k_{1}$ in

$$
\begin{gathered}
G_{1}=A_{210} \frac{\xi\left(2 \alpha \eta^{2}-h^{2}\right)}{4}-\eta A_{102} \alpha-A_{201} \frac{\eta\left(2 \alpha \xi^{2}+h^{2}\right)}{4}-k_{1} \frac{\eta\left(\xi^{2}+\eta^{2}\right)}{2} \\
G_{2}=-A_{210} \frac{\eta\left(2 \alpha \xi^{2}-h^{2}\right)}{4}+A_{102} \xi \alpha+A_{201} \frac{\xi\left(2 \alpha \xi^{2}-h^{2}\right)}{4}+k_{1} \frac{\xi\left(\xi^{2}+\eta^{2}\right)}{2} .
\end{gathered}
$$

\section{Translation symmetry}

The first potential is

$$
V_{T, y}=\frac{\alpha}{\xi^{2} \eta^{2}}=\frac{\alpha}{y^{2}}
$$

It admits 4 linearly independent constants of the motion associated with the constants $A_{012}, A_{030}, A_{111}, A_{210}$ and a Killing vector associated with $k_{1}$, with functions

$$
\begin{gathered}
G_{1}=\frac{\alpha\left(\eta^{4}+\xi^{4}\right) A_{210}}{2 \xi \eta^{2}}-\frac{\alpha(\eta-\xi)(\eta+\xi) A_{111}}{\xi \eta^{2}}+2 \frac{A_{012} \alpha}{\xi \eta^{2}}+k_{1} \xi \\
G_{2}=-\frac{\alpha\left(\eta^{4}+\xi^{4}\right) A_{210}}{2 \eta \xi^{2}}+\frac{\alpha(\eta-\xi)(\eta+\xi) A_{111}}{\eta \xi^{2}}-2 \frac{A_{012} \alpha}{\eta \xi^{2}}-k_{1} \eta .
\end{gathered}
$$

The potential

$$
V_{T, y, \hbar}=\frac{\hbar^{2}}{\xi^{2} \eta^{2}}=\frac{\hbar^{2}}{y^{2}}
$$

admits 8 linearly independent third-order constants of the motion associated with constants $A_{003}, A_{012}, A_{030}, A_{102}, A_{111}, A_{201}, A_{210}$, and $A_{300}$ as well as a Killing vector associated with the constant $k_{1}$. The $G$ 's are

$$
\begin{gathered}
G_{1}=\frac{h^{2}\left(3 \eta^{4}-\xi^{4}\right) A_{201}}{4 \eta \xi^{2}}+\frac{h^{2}\left(\eta^{4}+\xi^{4}\right) A_{210}}{2 \xi \eta^{2}}-\frac{h^{2}\left(3 \eta^{6}+5 \xi^{2} \eta^{4}+5 \xi^{4} \eta^{2}+3 \xi^{6}\right) A_{300}}{8 \eta \xi^{2}}+\xi k_{1} \\
G_{2}=\frac{h^{2}\left(3 \xi^{4}-\eta^{4}\right) A_{201}}{4 \xi \eta^{2}}-\frac{h^{2}\left(\eta^{4}+\xi^{4}\right) A_{210}}{2 \eta \xi^{2}}+\frac{h^{2}\left(3 \eta^{6}+5 \xi^{2} \eta^{4}+5 \xi^{4} \eta^{2}+3 \xi^{6}\right) A_{300}}{8 \xi \eta^{2}}-\eta k_{1} .
\end{gathered}
$$

Finally, the potential

$$
V_{T, x}=\alpha\left(\xi^{2}-\eta^{2}\right)=2 \alpha x
$$

admits four linearly independent third-order integrals associated with constants $A_{003}, A_{102}$, $A_{021}, A_{012}$ and a Killing vector associated with $k_{1}$, with

$$
\begin{gathered}
G_{1}=-\eta^{3} \xi^{2} A_{102} \alpha-2 \eta^{3} A_{021} \alpha+2 \eta^{2} \xi A_{012} \alpha+2 \eta \xi^{2} A_{021} \alpha+\eta k_{1} \\
G_{2}=-\xi^{3} A_{102} \alpha, \eta^{2}+2 \xi^{3} A_{021} \alpha+2 \eta A_{012} \xi^{2} \alpha-2 \xi A_{21} \alpha, \eta^{2}+\xi k_{1} .
\end{gathered}
$$




\section{CONCLUSIONS}

The main conclusion of this article is a negative one: all third-order integrals for potentials separating in parabolic coordinates in $E_{2}(\mathbb{R})$ are reducible. The corresponding potentials allow separation of variables in at least two coordinate systems and are already known to be quadratically superintegrable $\underline{\underline{83}}$. Thus $V_{1}(\underline{54})$ is a deformed anisotropic harmonic oscillator, separable also in Cartesian coordinates. The potential $V_{2}$ (55) is a deformed Coulomb potential, separable also in polar coordinates. The potential $V_{3}$ (56) is a different deformation of the Coulomb potential, separable in two different parabolic coordinate systems (with the directrix of the parabolas either along the $x$ or the $y$ axis). Since these potentials are second-order superintegrable, they are the same in classical and quantum mechanics.

This is in stark contrast with the results obtained in the case of potentials separating in Cartesian $\frac{10}{\underline{n}}$ or polar coordinates $\underline{36}$. There the results were much richer, mainly because of the role played by the linear compatibility condition (13).

Indeed, let us first consider a potential separating in Cartesian coordinates $V=W_{1}(x)+$ $W_{2}(y)$. The linear compatibility conditions for this potential, written in Cartesian coordinates is given by

$$
\begin{array}{r}
-F_{3} W_{1, x x x}+2\left(F_{2, y}-F_{3, x}\right) W_{1, x x}-\left(3 F_{1, y y}-2 F_{2, x y}+F_{3, x x}\right) W_{1, x}= \\
F_{2} W_{2, y y y}+\left(F_{2, y}-F_{3, x}\right) W_{2, y y}+\left(F_{2, y y}-2 F_{3, x y}+3 F_{4, x x}\right) W_{2 y}
\end{array}
$$

with

$$
\begin{aligned}
& F_{1}=A_{300} y^{3}+A_{210} y^{2}-A_{120} y+A_{030} \\
& F_{2}=3 A_{300} x y^{2}-2 A_{210} x y+A_{201} y^{2}+A_{120} x-A_{111} y+A_{021} \\
& F_{3}=-3 A_{300} x^{2} y+A_{210} x^{2}-2 A_{201} x y+A_{111} x-A_{102} y+A_{012} \\
& F_{4}=A_{300} x^{3}+A_{201} x^{2}+A_{102} x+A_{003} .
\end{aligned}
$$

Differentiating (64) twice with respect to $x$ gives two linear ODEs for $W_{1}$ :

$$
\begin{gathered}
\left(3 A_{300} x^{2}+2 A_{201} x+A_{102}\right) W_{1}^{(5)}+\left(36 A_{300} x+12 A_{201}\right) W_{1}^{(4)}+84 A_{300} W_{1}^{(3)}=0 \\
\left(-A_{111} x-A_{210} x^{2}-A_{012}\right) W_{1}^{(5)}+\left(-12 A_{210} x-6 A_{111}\right) W_{1}^{(4)}-28 A_{210} W_{1}^{(3)}=0 .
\end{gathered}
$$

Differentiating (64) twice with respect to $y$ gives two linear ODEs for $W_{2}$ :

$$
\left(3 A_{300} y^{2}-2 A_{210} y+A_{120}\right) W_{2}^{(5)}+\left(36 A_{300} y-12 A_{210}\right) W_{2}^{(4)}+90 A_{300} W_{1}^{(3)}=0
$$




$$
\left(A_{201} y^{2}-A_{111} y+A_{021}\right) W_{2}^{(5)}+\left(12 A_{201} y-6 A_{111}\right) W_{2}^{(4)}+30 A_{201} W_{2}^{(3)}=0 .
$$

Interesting potentials in quantum mechanics, involving elliptic functions or Painlevé trascendents are obtained if either of these pairs of linear equations are satisfied trivially, i.e. if the coefficients in (65+66) or (67-68) vanish identically. Both systems of equations (65+68) vanish identically if and only if the only non-zero coefficients $A_{j k \ell}$ are $A_{030}$ and $A_{003}$. The potential in this case is for instance $e^{10}$

$$
V(x, y)=\hbar^{2}\left[\omega_{1}^{2} P_{I}\left(\omega_{1} x\right)+\omega_{2}^{2} P_{I}\left(\omega_{1} y\right)\right],
$$

where $P_{I}$ is the first Painlevé transcendent $\underline{\underline{14}}$. If, for example, (67-68) are satisfied trivially but (65-66) are not, this leads to potentials of the form 10

$$
V(x, y)=a y+\left(2 \hbar^{2} b^{2}\right)^{1 / 3}\left[P_{I I}^{\prime}\left(-\left(\frac{4 b}{\hbar^{2}}\right)^{1 / 3} x, k\right)+P_{I I}^{2}\left(-\frac{4 b}{\hbar^{2}} x, k\right)\right],
$$

where $P_{I I}$ is the second Painlevé transcendent ${ }^{14}$.

Now, let us consider potentials allowing separation of variables in polar coordinates $\underline{36}$

$$
V=R(r)+\frac{1}{r^{2}} S(\theta) .
$$

The linear compatibility condition reduces to

$$
\begin{aligned}
0 & =r^{4} F_{3} R^{\prime \prime \prime}+\left(2 r^{4} F_{3, r}-2 r^{2} F_{2, \theta}+3 r\left(2 r^{2} F_{3}-F_{1}\right)\right) R^{\prime \prime} \\
& +\left(r^{4} F_{3, r r}+2 r^{2}\left(3 F_{3, r}+3 F_{3}-F_{2, r \theta}\right)-4 r F_{2, \theta}+3 F_{1, \theta \theta}\right) R^{\prime} \\
& +\frac{1}{r^{2}} F_{2} S^{\prime \prime \prime}-\frac{1}{r^{3}}\left(2 r^{3} F_{3, r}-2 r F_{2, \theta}+6 F_{1}\right) S^{\prime \prime} \\
& +\frac{1}{r^{3}}\left(3 r^{5} F_{4, r r}+6 r^{4} F_{4, r}-2 r^{3} F_{3, r \theta}+3 r^{2} F_{2, r}+r\left(F_{2, \theta \theta}-2 F_{2}\right)-12 F_{1, \theta}\right) S^{\prime} \\
& -\frac{1}{r^{3}}\left(2 r^{4} F_{3, r r}-12 r^{3} F_{3, r}+4 r^{2}\left(3 F_{3, r}-F_{2, r \theta}\right)+4 r F_{2, \theta}+6 F_{1, \theta \theta}+18 F_{1}\right) S
\end{aligned}
$$

where here the $F_{i}$ 's are given by

$$
\begin{aligned}
& F_{1}=A_{1} \cos 3 \theta+A_{2} \sin 3 \theta+A_{3} \cos \theta+A_{4} \sin \theta \\
& F_{2}=\frac{-3 A_{1} \sin 3 \theta+3 A_{2} \cos 3 \theta-A_{3} \cos \theta+A_{4} \sin \theta}{r}+B_{1} \cos 2 \theta+B_{2} \sin 2 \theta+B_{0} \\
& F_{3}=\frac{-3 A_{1} \cos 3 \theta-3 A_{2} \sin 3 \theta+A_{3} \cos \theta+A_{4} \sin \theta}{r^{2}}+\frac{-2 B_{1} \sin 2 \theta+2 B_{2} \cos 2 \theta}{r}+C_{1} \cos \theta+C_{2} \sin \theta \\
& F_{4}=\frac{A_{1} \sin 3 \theta-A_{2} \cos 3 \theta-A_{3} \sin \theta+A_{4} \cos \theta}{r^{3}}-\frac{B_{1} \cos 2 \theta+B_{2} \sin 2 \theta+B_{0}}{r^{2}}-\frac{C_{1} \sin \theta-C_{2} \cos \theta}{r}+D_{0},
\end{aligned}
$$

with

$$
\begin{array}{lll}
A_{1}=\frac{A_{030}-A_{012}}{4}, & A_{2}=\frac{A_{021}-A_{003}}{4}, & A_{3}=\frac{3 A_{030}+A_{012}}{4}, \\
A_{4}=\frac{3 A_{003}+A_{021}}{4}, & B_{1}=\frac{A_{120}-A_{102}}{2}, & B_{2}=\frac{A_{111}}{2}, \\
C_{1}=A_{210}, & C_{2}=A_{201}, & D_{0}=A_{300} .
\end{array}
$$


Equation (70) is satisfied trivially if all the $A_{j k \ell}=0$ except $A_{300}$. This leads to the potential $\underline{36}$

$$
V(r, \theta)=R(r)+\frac{\hbar^{2}}{r^{2}} P\left(\theta, t_{2}, t_{3}\right),
$$

where $R(r)$ is arbitrary and $P\left(\theta, t_{2}, t_{3}\right)$ is the Weierstrass elliptic function ${ }^{-}$. This potential allows a third-order integral, however it is algebraically related to the second-order one and the system is hence not superintegrable. If however we consider the subcase $R(r)=0$, Eq. (70) simplifies. It being satisfied trivially allows further constants in the integral to be nonzero, namely $A_{300}, A_{210}$ and $A_{201}$. This leads to a superintegrable potential expressed in terms of the sixth Painlevé transcendent $\underline{14}, P_{V I}(\sin \theta / 2)$.

In section III, we have shown that the linear compatibility condition (13) is never satisfied trivially for a potential separating in parabolic coordinates. This rules out any dependence of $W_{1}$ and $W_{2}$ on elliptic functions or Painlevé transcendents.

In addition to the real potentials presented in section $\mathrm{V}$, we have recovered a complex one (see Appendix B) which is known to be superintegrable $e^{22}$. It can be transformed into a real form; however, it will live on the pseudo-Euclidean plane $E(1,1)$ rather than the Euclidean one.

\section{ACKNOWLEDGMENTS}

We thank F. Tremblay for helpful discussions. SP acknowledges a postdoctoral fellowship provided by the Laboratory of Mathematical Physics of the Centre de Recherches Mathématiques, Université de Montréal. The research of PW is partially supported by the National Sciences and Engineering Research Council (NSERC) of Canada.

\section{Appendix A: System of linear ODEs for $W_{1}$ and $W_{2}$}

The linear compatibility condition (13), implies (by differentiation) two systems of 14 ODEs for $W_{1}$ and $W_{2}$, respectively. Those for $W_{1}$ are given below and those for $W_{2}$ can be

obtained from these under the interchange $\xi \rightarrow \eta$ and $A_{j k \ell} \rightarrow(-1)^{j+k} A_{j k \ell}$.

$0=A_{300}\left(\xi^{2} W_{1}^{(17)}+37 \xi W_{1}^{(16)}+323 W_{1}^{(15)}\right)$ 


$$
\begin{aligned}
& 0=A_{210}\left(\xi W_{1}^{(17)}+18 W_{1}^{(16)}\right) \\
& 0=A_{210}\left(11 \xi^{3} W_{1}^{(17)}+503 \xi^{2} W_{1}^{(16)}+7145 \xi W_{1}^{(15)}+31360 W_{1}^{(14)}\right) \\
& 0=-9 A_{300}\left(\xi^{4} W_{1}^{(17)}+65 \xi^{3} W_{1}^{(16)}+1485 \xi^{2} W_{1}^{(15)}+14070 \xi W_{1}^{(14)}+46410 W_{1}^{(13)}\right) \\
& +\left(A_{201} \xi^{2}-2 A_{120}\right) W_{1}^{(17)}-33 \xi A_{201} W_{1}^{(16)}-255 A_{201} W_{1}^{(15)} . \\
& 0=-\frac{45}{4} A_{300}\left(\xi^{6} W_{1}^{(17)}+93 \xi^{5} W_{1}^{(16)}+3375 \xi^{4} W_{1}^{(15)}+60900 \xi^{3} W_{1}^{(14)}+573300 \xi^{2} W_{1}^{(13)}\right. \\
& \left.+2653560 \xi W_{1}^{(12)}+4684680 W_{1}^{(11)}\right) \\
& -A_{201}\left(\frac{5 \xi^{4}}{2} W_{1}^{(17)}+\frac{301 \xi^{3}}{2} W_{1}^{(16)}+\frac{6345 \xi^{2}}{2} W_{1}^{(15)}+27615 \xi W_{1}^{(14)}+83265 W_{1}^{(13)}\right) \\
& -A_{120}\left(6 \xi^{2} W_{1}^{(17)}+169 \xi W_{1}^{(16)}+1109 W_{1}^{(15)}\right) \\
& +\left(\xi^{2} A_{102}+2 A_{021}\right) W_{1}{ }^{17}+33 \xi A_{102} W_{1}{ }^{(16)}+255 A_{102} W_{1}{ }^{(15)} . \\
& 0=\frac{-15}{2} A_{300}\left(\xi^{8} W_{1}^{(17)}+121 \xi^{7} W_{1}^{(16)}+5993 \xi^{6} W_{1}^{(15)}+157962 \xi^{5} W_{1}^{(14)}+2410590 \xi^{4} W_{1}^{(13)}\right. \\
& \left.+21676200 \xi^{3} W_{1}^{(12)}+111351240 \xi^{2} W_{1}^{(11)}+296215920 \xi W_{1}^{(10)}+309188880 W_{1}^{(9)}\right) \\
& -A_{201}\left(\frac{5 \xi^{6}}{2} W_{1}^{(17)}+\frac{437 \xi^{5}}{2} W_{1}^{(16)}+\frac{14831 \xi^{4}}{2} W_{1}^{(15)}+124418 \xi^{3} W_{1}^{(14)}\right. \\
& \left.+1081626 \xi^{2} W_{1}^{(13)}+4585308 \xi W_{1}^{(12)}+7339332 W_{1}^{(11)}\right) \\
& -A_{120}\left(7 \xi^{4} W_{1}^{(17)}+388 \xi^{3} W_{1}^{(16)}+7464 \xi^{2} W_{1}^{15}+58632 \xi W_{1}^{(14)}+157248 W_{1}^{(13)}\right) \\
& +2 A_{102}\left(\xi^{4} W_{1}^{(17)}+58 \xi^{3} W_{1}^{(16)}+1170 \xi^{2} W_{1}^{(15)}+9660 \xi W_{1}^{(14)}+27300 W_{1}^{(13)}\right) \\
& +A_{021}\left(\xi^{2} W_{1}^{(17)}-11 \xi W_{1}^{(16)}-409 W_{1}^{(15)}\right) \\
& +A_{003}\left(3 \xi^{2} W_{1}^{(17)}+111 \xi W_{1}^{(16)}+969 W_{1}^{(15)}\right) . \\
& 0=A_{210}\left(\frac{25 \xi^{5}}{4} W_{1}^{(17)}+\frac{1839 \xi^{4}}{4} W_{1}^{(16)}+\frac{50451 \xi^{3}}{4} W_{1}^{(15)}+\frac{320901 \xi^{2}}{2} W_{1}{ }^{14}+\frac{1881243 \xi}{2} W_{1}{ }^{(13)}\right. \\
& \left.+2018016 W_{1}^{(12)}\right) \\
& +\left(3 \xi A_{030}+1 / 2 \xi^{3} A_{111}-2 \xi A_{012}\right) W_{1}^{17}+\left(54 A_{030}-36 A_{012}+\frac{51}{2} \xi^{2} A_{111}\right) W_{1}{ }^{(16)} \\
& +\frac{813}{2} W_{1}^{(15)} \xi A_{111}+2016 W_{1}^{(14)} A_{111} \text {. } \\
& 0=\frac{1}{4} A_{210}\left(25 W_{1}^{(17)} \xi^{5}+1839 W_{1}^{(16)} \xi^{4}+50451 W_{1}^{(15)} \xi^{3}+641802 W_{1}^{(14)} \xi^{2}+3762486 W_{1}^{(13)} \xi\right. \\
& \left.+8072064 W_{1}^{(12)}\right) \\
& +\frac{1}{2} A_{111}\left(W_{1}^{(17)} \xi^{3}+51 W_{1}^{(16)} \xi^{2}+813 W_{1}^{(15)} \xi+4032 W_{1}^{(14)}\right) \\
& +3 A_{030}\left(W_{1}^{(17)} \xi+18 W_{1}^{(16)}\right)-2 A_{012}\left(W_{1}^{(17)} \xi+18 W_{1}^{(16)}\right) .
\end{aligned}
$$




$$
\begin{aligned}
& 0=\frac{1}{2} A_{210}\left(15 W_{1}^{(17)} \xi^{7}+1523 W_{1}^{(16)} \xi^{6}+61761 W_{1}^{(15)} \xi^{5}+1289820 W_{1}^{(14)} \xi^{4}+14889420 W_{1}^{(13)} \xi^{3}\right. \\
& \left.+94316040 W_{1}^{(12)} \xi^{2}+300900600 W_{1}^{(11)} \xi+369008640 W_{1}^{(10)}\right) \\
& +2 A_{111}\left(W_{1}^{(17)} \xi^{5}+76 W_{1}^{(16)} \xi^{4}+2156 W_{1}^{(15)} \xi^{3}+28392 W_{1}^{(14)} \xi^{2}+172536 W_{1}^{(13} \xi\right. \\
& \left.+384384 W_{1}^{(12)}\right) \\
& -A_{012}\left(5 W_{1}^{(17)} \xi^{3}+173 W_{1}^{(16)} \xi^{2}+1475 W_{1}^{(15)} \xi+1024 W_{1}^{(14)}\right) \\
& 3 A_{030}\left(3 W_{1}^{(17)} \xi^{3}+115 W_{1}^{(16)} \xi^{2}+1249 W_{1}^{(15)} \xi+3392 W_{1}^{(14)}\right) \text {. } \\
& 0=-\frac{9}{4} A_{300}\left(W_{1}^{(17)} \xi^{12}+177 W_{1}^{(16)} \xi^{11}+13413 W_{1}^{(15)} \xi^{10}+572670 W_{1}^{(14)} \xi^{9}+15257970 W_{1}^{(13)} \xi^{8}\right. \\
& +265552560 W_{1}^{(12)} \xi^{7}+3072429360 W_{1}^{(11)} \xi^{6}+23597814240 W_{1}^{(10)} \xi^{5} \\
& +118118800800 W_{1}{ }^{(9)} \xi^{4}+370767196800 W_{1}{ }^{(8)} \xi^{3}+681080400000 W_{1}{ }^{(7)} \xi^{2} \\
& \left.+642939897600 W_{1}{ }^{(6)} \xi+228843014400 W_{1}{ }^{(5)}\right) \\
& -\frac{1}{4} A_{201}\left(5 W_{1}^{(17)} \xi^{10}+709 W_{1}^{(16)} \xi^{9}+42051 W_{1}^{(15)} \xi^{8}+1365672 W_{1}^{(14)} \xi^{7}\right. \\
& +26708136 W_{1}^{(13)} \xi^{6}+325909584 W_{1}^{(12)} \xi^{5}+2487204720 W_{1}^{(11)} \xi^{4} \\
& +11568997440 W_{1}^{(10)} \xi^{3}+30849698880 W_{1}^{(9)} \xi^{2} \\
& \left.+41565363840 W_{1}{ }^{(8)} \xi+20704844160 W_{1}{ }^{(7)}\right) \\
& -\frac{1}{2} A_{120}\left(9 W_{1}^{(17)} \xi^{8}+976 W_{1}^{(16)} \xi^{7}+42728 W_{1}^{(15)} \xi^{6}+978432 W_{1}^{(14)} \xi^{5}+12689040 W_{1}^{(13)} \xi^{4}\right. \\
& \left.+94174080 W_{1}^{(12)} \xi^{3}+383423040 W_{1}^{(11)} \xi^{2}+761080320 W_{1}^{(10)} \xi+536215680 W_{1}{ }^{(9)}\right) \\
& +2 A_{102}\left(W_{1}^{(17)} \xi^{8}+108 W_{1}^{(16)} \xi^{7}+4704 W_{1}^{(15)} \xi^{6}+107016 W_{1}^{(14)} \xi^{5}+1375920 W_{1}^{(13)} \xi^{4}\right. \\
& \left.+10090080 W_{1}^{(12)} \xi^{3}+40360320 W_{1}^{(11)} \xi^{2}+77837760 W_{1}^{(10)} \xi+51891840 W_{1}^{(9)}\right) \\
& -A_{021}\left(5 W_{1}^{(17)} \xi^{6}+393 W_{1}^{(16)} \xi^{5}+11511 W_{1} \xi^{(15)}+157140 W_{1}^{(14)} \xi^{3}+1016820 W_{1}^{(13)} \xi^{2}\right. \\
& \left.+2784600 W_{1}^{(12)} \xi+2325960 W_{1}^{(11)}\right) \\
& +9 A_{003}\left(W_{1}^{(17)} \xi^{6}+77 W_{1}^{(16)} \xi^{5}+2223 W_{1}^{(15)} \xi^{4}+30180 W_{1}^{(14)} \xi^{3}+196980 W_{1}^{(13)} \xi^{2}\right. \\
& \left.+556920 W_{1}^{(12)} \xi+491400 W_{1}^{(11)}\right) \text {. }
\end{aligned}
$$




$$
\begin{aligned}
0= & \frac{45}{8} A_{300}\left(\xi^{10} W_{1}^{(17)}+149 \xi^{9} W_{1}^{(16)}+9339 \xi^{8} W_{1}^{(15)}+322728 \xi^{7} W_{1}^{(14)}\right. \\
& +6772584 \xi^{6} W_{1}^{(13)}+89618256 \xi^{5} W_{1}^{(12)}+751710960 \xi^{4} W_{1}^{(11)}+3912068160 \xi^{3} W_{1}^{(10)} \\
& \left.+11961069120 \xi^{2} W_{1}^{(9)}+19148088960 \xi W_{1}^{(8)}+11987015040 W_{1}^{(7)}\right) \\
- & A_{201}\left(\frac{5 \xi^{8}}{2} W_{1}^{(17)}+\frac{573 \xi^{7}}{2} W_{1}^{(16)}+\frac{26733 \xi^{6}}{2} W_{1}^{(15)}+329721 \xi^{5} W_{1}^{(14)}+4672395 \xi^{4} W_{1}^{(13)}\right. \\
& \left.+38640420 \xi^{3} W_{1}^{(12)}+180360180 \xi^{2} W_{1}^{(11)}+429188760 \xi W_{1}^{(10)}+392432040 W_{1}^{(9)}\right) \\
- & A_{120}\left(8 \xi^{6} W_{1}^{(17)}+657 \xi^{5} W_{1}^{(16)}+20769 \xi^{4} W_{1}^{(15)}+320964 \xi^{3} W_{1}^{(14)}+2532348 \xi^{2} W_{1}^{(13)}\right. \\
& \left.+9546264 \xi W_{1}^{(12)}+13189176 W_{1}^{(11)}\right) \\
+ & 3 A_{102}\left(\xi^{6} W_{1}^{(17)}+83 \xi^{5} W_{1}^{(16)}+2653 \xi^{4} W_{1}^{(15)}+41468 \xi^{3} W_{1}^{(14)}\right. \\
& \left.+330876 \xi^{2} W_{1}^{(13)}+1260168 \xi W_{1}^{(12)}+1753752 W_{1}^{(11)}\right) \\
- & 3 A_{021}\left(\xi^{4} W_{1}^{(17)}+71 \xi^{3} W_{1}^{(16)}+1583 \xi^{2} W_{1}^{(15)}+12858 \xi W_{1}^{(14)}+29022 W_{1}^{(13)}\right) \\
+ & 9 A_{003}\left(\xi^{4} W_{1}^{(17)}+57 \xi^{3} W_{1}^{(16)}+1093 \xi^{2} W_{1}^{(15)}+8070 \xi W_{1}^{(14)}+17850 W_{1}^{(13)}\right) .
\end{aligned}
$$




$$
\begin{aligned}
& 0=\frac{-8}{3} A_{300}\left(\xi^{14} W_{1}^{(17)}+205 \xi^{13} W_{1}^{(16)}+18215 \xi^{(12)} W_{1}^{(15)}+925260 \xi^{11} W_{1}^{(14)}\right. \\
& +29849820 \xi^{10} W_{1}^{(13)}+642762120 \xi^{9} W_{1}^{(12)}+9454044600 \xi^{8} W_{1}^{(11)}+95610715200 \xi^{7} W_{1}^{10} \\
& +660712852800 \xi^{6} W_{1}^{(9)}+3062396937600 \xi^{5} W_{1}^{(8)}+9210023222400 \xi^{4} W_{1}{ }^{(7)} \\
& +17036091072000 \xi^{3} W_{1}^{(6)}+17762576832000 \xi^{2} W_{1}{ }^{(5)} \\
& \left.+8935774848000 \xi W_{1}{ }^{(4)}+1525620096000 W_{1}{ }^{(3)}\right) \\
& -\frac{1}{4} A_{201}\left(W_{1}^{(17)} \xi^{12}+169 W_{1}^{(16)} \xi^{11}+12157 W_{1}^{(15)} \xi^{10}+489230 W_{1}^{(14)} \xi^{9}+12178530 W_{1}^{(13)} \xi^{8}\right. \\
& +195839280 W_{1}^{(12)} \xi^{7}+2063421360 W_{1}^{(11)} \xi^{6}+14153499360 W_{1}^{(10)} \xi^{5} \\
& +61556695200 W_{1}{ }^{(9)} \xi^{4}+161124163200 W_{1}{ }^{(8)} \xi^{3}+230659228800 W_{1}{ }^{(7)} \xi^{2} \\
& \left.+148929580800 W_{1}^{(6)} \xi+25427001600 W_{1}^{(5)}\right) \\
& -\frac{1}{2} A_{120}\left(2 W_{1}^{(17)} \xi^{10}+269 W_{1}^{(16)} \xi^{9}+15009 W_{1}^{(15)} \xi^{8}+453768 W_{1}^{(14)} \xi^{7}+8148504 W_{1}^{\left(13 \gamma g^{6} 12\right)}\right. \\
& +89618256 W_{1}^{(12)} \xi^{5}+600359760 W_{1}^{(11)} \xi^{4}+2355312960 W_{1}^{(10)} \xi^{3}+4955670720 W_{1}^{(9)} \xi^{2} \\
& \left.+4618373760 W_{1}^{(8)} \xi+1089728640 W_{1}^{(7)}\right) \\
& +\frac{1}{2} A_{102}\left(W_{1}^{(17)} \xi^{10}+133 W_{1}^{(16)} \xi^{9}+7323 W_{1}^{(15)} \xi^{8}+217896 W_{1}^{(14)} \xi^{7}+3837288 W_{1}^{(13)} \xi^{6}\right. \\
& +41185872 W_{1}^{(12)} \xi^{5}+267387120 W_{1}^{(11)} \xi^{4}+1006125120 W_{1}^{(10)} \xi^{3}+1997835840 W_{1}^{(9)} \xi^{2} \\
& \left.+1712430720 W_{1}{ }^{(8)} \xi+363242880 W_{1}^{(7)}\right) \\
& -A_{021}\left(2 W_{1}^{(17)} \xi^{8}+191 W_{1}^{(16)} \xi^{7}+7171 W_{1}^{(15)} \xi^{6}+135846 W_{1}^{(14)} \xi^{5}+1385370 W_{1}^{(13)} \xi^{4}\right. \\
& \left.+7502040 W_{1}^{(12)} \xi^{3}+19819800 W_{1}^{(11)} \xi^{2}+20900880 W_{1}^{(10)} \xi+5045040 W_{1}^{(9)}\right) \\
& +3 A_{003}\left(W_{1}^{(17)} \xi^{8}+97 W_{1}^{(16)} \xi^{7}+3713 W_{1}^{(15)} \xi^{6}+72090 W_{1}^{(14)} \xi^{5}+759150 W_{1}^{(13)} \xi^{4}\right. \\
& \left.+4291560 W_{1}^{(12)} \xi^{3}+12022920 W_{1}^{(11)} \xi^{2}+13693680 W_{1}^{(10)} \xi+3603600 W_{1}^{(9)}\right) . \\
& 0=\frac{1}{2} A_{210}\left(10 W_{1}^{(17)} \xi^{9}+1297 W_{1}^{(16)} \xi^{8}+69655 W_{1}^{(15)} \xi^{7}+2022230 W_{1}^{(14)} \xi^{6}+34761090 W_{1}^{(13)} \xi^{5}\right. \\
& +364236600 W_{1}^{(12)} \xi^{4}+2306424120 W_{1}^{(11)} \xi^{3}+8421613200 W_{1}^{(10)} \xi^{2} \\
& \left.+15881065200 W_{1}{ }^{(9)} \xi+11589177600 W_{1}{ }^{(8)}\right) \\
& +3 A_{111}\left(W_{1}^{(17)} \xi^{7}+101 W_{1}^{(16)} \xi^{6}+4067 W_{1}^{(15)} \xi^{5}+84140 W_{1}^{(14)} \xi^{4}+959140 W_{1}^{(13)} \xi^{3}\right. \\
& \left.+5973240 W_{1}^{(12)} \xi^{2}+18618600 W_{1}^{(11)} \xi+22102080 W_{1}^{(10)}\right) \\
& +9 A_{030}\left(W_{1}^{(17)} \xi^{5}+59 W_{1}^{(16)} \xi^{4}+1227 W_{1}^{(15)} \xi^{3}+10946 W_{1}^{(14)} \xi^{2}+40838 W_{1}^{(13)} \xi\right. \\
& \left.+53872 W_{1}^{(12)}\right) \\
& -3 A_{012}\left(W_{1}^{(17)} \xi^{5}+41 W_{1}^{(16)} \xi^{4}+273 W_{1}^{(15)} \xi^{3}-6146 W_{1}^{(14)} \xi^{2}-78638 W_{1}^{(13)} \xi\right. \\
& \left.-206752 W_{1}^{(12)}\right) \text {. }
\end{aligned}
$$




$$
\begin{aligned}
0=\frac{1}{4} & A_{210}\left(7 W_{1}^{(17)} \xi^{11}+1107 W_{1}^{(16)} \xi^{10}+74133 W_{1}^{(15)} \xi^{9}+2760408 W_{1}^{(14)} \xi^{8}\right. \\
& +63115416 W_{1}^{(13)} \xi^{7}+923792688 W_{1}^{(12)} \xi^{6}+8757180432 W_{1}^{(11)} \xi^{5}+53215081920 W_{1}^{(10)} \xi^{4} \\
& +200587907520 W_{1}^{(9)} \xi^{3}+439575776640 W_{1}^{(8)} \xi^{2}+493647073920 W_{1}^{(7)} \xi \\
& \left.+209227898880 W_{1}^{(6)}\right) \\
+ & 2 A_{111}\left(W_{1}^{(17)} \xi^{9}+126 W_{1}^{(16)} \xi^{8}+6546 W_{1}^{(15)} \xi^{7}+182868 W_{1}^{(14)} \xi^{6}+3004092 W_{1}^{(13)} \xi^{5}\right. \\
& +29811600 W_{1}^{(12)} \xi^{4}+176576400 W_{1}^{(11)} \xi^{3}+592431840 W_{1}^{(10)} \xi^{2}+998917920 W_{1}^{(9)} \xi \quad(\mathrm{A} 14) \\
& \left.+622702080 W_{1}^{(8)}\right) \\
+ & 3 A_{030}\left(W_{1}^{(17)} \xi^{7}+81 W_{1}^{(16)} \xi^{6}+2535 W_{1}^{(15)} \xi^{5}+39444 W_{1}^{(14)} \xi^{4}+329364 W_{1}^{(13)} \xi^{3}\right. \\
& \left.+1500408 W_{1}^{(12)} \xi^{2}+3583944 W_{1}^{(11)} \xi+3459456 W_{1}^{(10)}\right) \\
+ & A_{012}\left(W_{1}^{(17)} \xi^{7}+129 W_{1}^{(16)} \xi^{6}+6027 W_{1}^{(15)} \xi^{5}+134484 W_{1}^{(14)} \xi^{4}+1544004 W_{1}^{(13)} \xi^{3}\right. \\
& \left.+8969688 W_{1}^{(12)} \xi^{2}+23633064 W_{1}^{(11)} \xi+20756736 W_{1}^{(10)}\right) .
\end{aligned}
$$

\section{Appendix B: Complex potentials and superintegrable systems in $E(1,1)$ admitting separation in parabolic coordinates}

As a byproduct of this study, we have obtained a complex potential in $E_{2}$ that can be viewed as real potential in the pseudo-Euclidean place $E(1,1)$. This is the only complex

potential in Ref. 22 which is separable in parabolic coordinates and does not admit a Killing vector. We also give the subcase of this potential which admits a Killing vector and its third-order integrals.

\section{System admitting a single third-order integral: $V_{4}$}

The following potential

$$
\begin{aligned}
V_{4} & =-\alpha\left(\xi^{2}-\eta^{2}\right)+\beta \frac{\left(\xi^{2}+i \xi \eta-\eta^{2}\right)}{\xi+i \eta}+\frac{\gamma}{\xi+i \eta}, \\
& =2 \alpha x-\frac{\beta(2 x+i y)}{\sqrt{x+i y}}-\frac{\gamma}{\sqrt{x+i y}} \\
& =2 \alpha r \cos (\theta)-\frac{r(2 \cos \theta+i \sin \theta) \beta+\gamma}{\sqrt{r(\cos \theta+i \sin \theta)}}
\end{aligned}
$$


admits one third-order integral with $A_{3}=-A_{021}, A_{012}=2 i A_{021}$ and the remained $A_{j k \ell}=0$. The functions $G_{1}$ and $G_{2}$ are given by

$$
\begin{gathered}
G_{1}=i\left(-2 i \eta \alpha \xi^{2}+4 \xi \alpha \eta^{2}-2 i \eta \xi \beta+2 i \alpha \eta^{3}+\eta^{2} \beta+\gamma\right) A_{021} \\
G_{2}=\left(-2 \xi \alpha \eta^{2}+4 i \eta \alpha \xi^{2}+2 i \eta \xi \beta+2 \xi^{3} \alpha+\beta \xi^{2}-\gamma\right) A_{021}
\end{gathered}
$$

Putting $i y=t$, we obtain a superintegrable system in $E(1,1)$; namely,

$$
H=\frac{1}{2}\left(p_{x}^{2}-p_{t}^{2}\right)+2 \alpha x-\frac{\beta(2 x+t)}{\sqrt{x+t}}-\frac{\gamma}{\sqrt{x+t}} .
$$

\section{System admitting group symmetry}

A special case of the previous potential admits a Killing vector. The potential is:

$$
V_{L}=\frac{\gamma}{\xi+i \eta}
$$

The third-order integral is defined by the following non-zero $A_{j k \ell}$, along with an arbitrary constant $k_{1}$ which is the coefficient of the Killing vector

$$
\begin{gathered}
A_{003}=A_{030}=\frac{A_{021}+2 i A_{012}}{3}, A_{120}=-i A_{111}+A_{102}, A_{102}, A_{111}, A_{021}, A_{012} \\
G_{1}=\gamma A_{012}+i \gamma A_{021}+i \gamma(\xi+i \eta)^{2} A_{102}-\frac{\gamma}{2}\left(\xi^{2}-\eta^{2}\right) A_{111}+k_{1}(\xi+i \eta) \\
G_{2}=i \gamma A_{012}+\gamma A_{021}+\frac{\gamma}{2}(\xi+i \eta)^{2} A_{102}-\frac{i \gamma}{2}\left(\xi^{2}-\eta^{2}\right) A_{111}+i k_{1}(\xi+i \eta) .
\end{gathered}
$$

\section{REFERENCES}

${ }^{1}$ Bargmann, V., "Zur theorie des Wasserstoffatoms," Zeitschrift für Physik 99, 576-582 (1936).

${ }^{2}$ Bertrand, J., "Thèorème relatif au mouvement d'un point attiré vers un centre fixe," C. R. Acad. Sci. 77, 849 - 853 (1873).

${ }^{3}$ Byrd, P. and Friedman, M., Handbook of Elliptic Integrals for Engineers and Students (Springer-Verlag, New York, 1972).

${ }^{4}$ Chanu, C., Degiovanni, L., and Rastelli, G., "Polynomial constants of motion for Calogerotype systems in three dimensions," J. Math. Phys. 52, 032903 (2011). 
${ }^{5}$ Drach, J., "Sur l'intégration logique des équations de la dynamique á deux variables: Forces conservatives. Intégrales cubiques. Mouvements dans le plan.” C.R. Acad. Sci. 200, 22-26 (1935).

${ }^{6}$ Evans, N. W., "Superintegrability in classical mechanics," Phys. Rev. A. 41, 5668-70 (1990).

${ }^{7}$ Fock, V., "Zur theorie des Wasserstoffatoms," Zeitschrift für Physik 98, 145-154 (1935).

${ }^{8}$ Fris, I., Mandrosov, V., Smorodinsky, J. A., Uhlír, M., and Winternitz, P., "On higher symmetries in quantum mechanics," Phys. Lett. 16, 354-356 (1965).

${ }^{9}$ Goldstein, H., Poole, C. P., and Safko, J. L., Classical Mechanics, 3rd ed. (Addison Wesley, 2001).

${ }^{10}$ Gravel, S., "Hamiltonians separable in Cartesian coordinates and third-order integrals of motion." J. Math Phys. 45, 1003-1019 (2004).

${ }^{11}$ Gravel, S. and Winternitz, P., "Superintegrability with third-order integrals in quantum and classical mechanics." J. Math. Phys. 46, 5902 (2002).

${ }^{12}$ Hietarinta, J., "Classical versus quantum integrability," J. Math. Phys. 25, 1833-1840 (1984).

${ }^{13}$ Hietarinta, J. and Grammaticos, B., "On the $\hbar^{2}$-correction terms in quantum mechanics," J. Phys. A 22, 1315-1322 (1989).

${ }^{14}$ Ince, E., Ordinary Differential Equations (Dover, New York, 1972).

${ }^{15}$ Jauch, J. and Hill, E., "On the problem of degeneracy in quantum mechanics," Phys. Rev 57, 641-645 (1940).

${ }^{16}$ Kalnins, E. G., Kress, J., Miller Jr., W., and Winternitz, P., "Superintegrable systems in Darboux spaces," J. Math. Phys. 44, 5811-5848 (2003).

${ }^{17}$ Kalnins, E. G., Kress, J., and Miller Jr., W., "Second order superintegrable systems in conformally flat spaces. i: 2d classical structure theory," J. Math. Phys. 46, 053509 (2005).

${ }^{18}$ Kalnins, E. G., Kress, J., and Miller Jr., W., "Second order superintegrable systems in conformally flat spaces. v. 2d and 3d quantum systems," J. Math Phys. 47, 093501 (2006).

${ }^{19}$ Kalnins, E. G., Kress, J., and Miller Jr., W., "A recurrence relation approach to higher order quantum superintegrability," SIGMA 7, 031 (2011).

${ }^{20}$ Kalnins, E. G., Kress, J. M., and Miller Jr., W., "Families of classical superintegrable systems," J. Phys. A 43, 092001 (2010). 
${ }^{21}$ Kalnins, E. G., Kress, J. M., and Miller Jr., W., "Superintegrability and higher order constants for quantum systems," J. Phys. A 43, 265205 (2010).

${ }^{22}$ Kalnins, E. G., Miller Jr. , W., and Pogosyan, G. S., "Completeness of multiseparable superintegrability in two dimensions," Phys. Atomic Nuclei 6, 1033-1035 (2002).

${ }^{23}$ Maciejewski, A. J., Przybylska, M., and Yoshida, H., "Necessary conditions for classical super-integrability of a certain family of potentials in constant curvature spaces," J. Phys. A 43, 382001 (2010).

${ }^{24}$ Makarov, A. A., Smorodinsky, J. A., Valiev, K., and Winternitz, P., "A systematic search for nonrelativistic systems with dynamical symmetries," Il Nuovo Cimento A 52, 10611084 (1967).

${ }^{25}$ Marquette, I., "Superintegrability with third order integrals of motion, cubic algebras, and supersymmetric quantum mechanics. ii. Painlevé transcendent potentials," J. Math. Phys. 50, 095202 (2009).

${ }^{26}$ Marquette, I., "Supersymmetry as a method of obtaining new superintegrable systems with higher order integrals of motion," J. Math. Phys. 50, 122102 (2009).

${ }^{27}$ Marquette, I. and Winternitz, P., "Superintegrable systems with third order integrals of motion," J. Phys. A , 303031-41 (2008).

${ }^{28}$ Moshinsky, M. and Smirnov, Y., The Harmonic Oscillator in Modern Physics, Vol. 9 (Amsterdam, Harwood Academic Publishers, 1996).

${ }^{29}$ Post, S. and Winternitz, P., "An infinite family of superintegrable deformations of the Coulomb potential," J. Phys. A. 42, 222001 (2010).

${ }^{30}$ Quesne, C., "Superintegrability of the Tremblay-Turbiner-Winternitz quantum Hamiltonian on a plane for odd k," J.Phys. A. 43, 082001 (2010).

${ }^{31}$ Rañada, M., "Superintegrable $n=2$ systems, quadratic constants of motion, and potentials of Drach," J. Math. Phys. 38, 4165-4178 (1997).

${ }^{32}$ Rañada, M., "Master symmetries, non-Hamiltonian symmetries and superintegrability of the generalized Smorodinsky-Winternitz," J. Phys. A 45, 145204 (2012).

${ }^{33}$ Tempesta, P., Winternitz, P., Miller, W., and Pogosyan, G., eds., Superintegrability in Classical and Quantum Systems, CRM Proceedings and Lecture Notes, Vol. 37 (AMS, 2004).

${ }^{34}$ Tremblay, F., Turbiner, A., and Winternitz, P., "An infinite family of solvable and integrable quantum systems on a plane," J. Phys. A 42, 242001 (2009). 
${ }^{35}$ Tremblay, F., Turbiner, A. V., and Winternitz, P., "Periodic orbits for an infinite family of classical superintegrable systems," J. Phys. A 43, 051202 (2010).

${ }^{36}$ Tremblay, F. and Winternitz, P., "Third order superintegrable systems separating in polar coordinates," J. Phys. A 43, 175206 (2010).

${ }^{37}$ Tsiganov, A., "Canonical transformations of the extended phase space, Toda lattices and Stäckel family of integrable systems," J. Phys. A. 33, 4169-4182 (1999).

${ }^{38}$ Winternitz, P., Smorodinsky, J. A., Uhlir, M., and Fris, I., "Symmetry groups in classical and quantum mechanics," Yad Fiz 4, 625-635 (1966), (1966 Sov. J. Nucl. Phys. 4 444-50). 